\title{
Que faire de (la mort de) Pompée ? Analyse poétique et politique d'une tragédie double
}

\author{
Guðrún Kristinsdóttir-Urfalino (Université d'Islande, Islande - Université \\ Sorbonne-Nouvelle - Paris 3, France)
}

\begin{abstract}
This article proposes a double analysis, poetic and political, of the tragedy La mort de Pompée (The Death of Pompey, 1644) by Pierre Corneille. It shows the bold construction of Corneille who crosses two intrigues: an Egyptian one (what to do with the arrival of Pompey?) and a Roman one (what to do with the death of Pompey?). The French poet lends to Caesar, faced with the head of Pompey, a double reaction, worked by the tension between the joy of the defeat of his adversary and the magnanimity that he must manifest to hope to restore civil peace. By inventing a confrontation, absent from the historical sources, between Caesar and Cornelia, he introduces the question of the conditions of the end of the civil war. It thus appears that the poetics of the play unfolds a political thought. Corneille highlights the threat that imperial conquest outside Rome poses for civil peace.
\end{abstract}

Keywords: La mort de Pompée, Pierre Corneille, French tragedy, poetics, Roman civil war

\section{Introduction}

La critique cornélienne a longuement opposé l'analyse poétique des tragédies de Corneille à leur analyse politique. Ainsi parmi les commentateurs les plus connus, Georges Forestier (2004) rejette l'analyse politique de Corneille au profit d'une analyse génétique de la poétique de son œuvre. D'autres, tels que Michel Prigent (2008) et Georges Couton (1980), voient dans les pièces de Corneille le reflet des conflits politiques du dix-septième siècle. Il est vrai que ces deux derniers ont sans doute trop cherché à retrouver dans ces tragédies des éléments de l'actualité politique de leur auteur. Mais indépendamment de cette actualité, la tragédie traite des grands conflits, tensions et souffrances issus des affaires humaines, d'où le politique surgit nécessairement, surtout si on l'entend dans un sens large. D'ailleurs, Hélène Merlin-Kajman $(1994,2000)$ a montré comment les spectateurs et les lecteurs du dixseptième siècle pouvaient penser le pouvoir et la chose publique par le biais de la littérature et du théâtre.

Nous avons donc pris le parti de conjuguer une analyse poétique et une analyse politique des pièces de Corneille. Cette démarche est particulièrement fructueuse dans le cas de La mort de Pompée dont la structure originale a pu dérouter la critique quand elle s'y est intéressée. Une étude alliant une lecture poétique et une lecture politique révèle au contraire une structure complexe autant que serrée.

Après une mise en perspective des principales études antérieures de la pièce (I), nous analyserons les articulations entre la poétique et la politique dans La mort de Pompée en quatre points : une construction de la pièce en deux tragédies reflétant les enjeux politiques 
(II) ; le jeu des perspectives historiques et temporelles (III) ; la (double) réaction de César face à la tête coupée de Pompée (IV); et la présence de Cornélie, personnage ajouté à la pièce par Corneille, permettant un face-à-face des deux parties romaines (V).

\section{Que faire de La mort de Pompée ?}

La mort de Pompée reste une pièce peu étudiée alors qu'elle était considérée au dixseptième siècle comme un chef-d'œuvre. Les études antérieures ont manifesté l'embarras des commentateurs, embarras relatif au genre de la pièce, à l'identité des protagonistes principaux et au caractère de César.

Le statut de la pièce a intrigué la critique qui s'est demandé s'il s'agissait bien d'une tragédie $^{1}$; s'il s'agissait d'une tragédie à fin heureuse ${ }^{2}$ ou d'une œuvre de transition dans laquelle l'intention de l'auteur est difficile à saisir, voire d'une pastorale, du moins en ce qui concerne le duo César-Cléopâtre ${ }^{3}$. La construction de la tragédie et son titre ont déconcerté la critique qui l'ont jugée incohérente ou tout au moins s'écartant largement des chefs-d'œuvre qui l'ont précédée 4 .

Le personnage de Pompée étant absent de la pièce, s'est posée la question de savoir quel était le véritable héros de la pièce. Marc Fumaroli a privilégié un axe d'analyse formé par le couple Pompée/César en en faisant un épisode de la guerre civile que prolongeraient leurs successeurs. Selon lui, Pompée et César incarnent deux figures de la magnanimité, présentes dès l'Éthique à Nicomaque d'Aristote et transmises par la tradition rhétorique et humaniste jusqu'à Corneille ${ }^{5}$; Pompée, la forme contemplative de la magnanimité, et César, la forme active, tendanciellement furieuse et tyrannique. Forestier voit l'action centrale de la pièce dans la confrontation entre César et Ptolomée. Il s'appuie sur Corneille lui-même pour justifier ce choix. Dans son Discours du poème dramatique, Corneille résume cette confrontation pour illustrer la nécessité de faire en sorte que l'action dramatique soit achevée devant le spectateur :

Ptolomée, périssant dans un combat avec ses ministres, laisse Cléopâtre en paisible possession du royaume dont elle demandait la moitié, et César hors de péril ; l'auditeur n'a plus rien à demander et sort satisfait, parce que l'action est complète ${ }^{6}$.

Le personnage de César a également fait l'objet d'interprétations opposées. Fumaroli estime que Corneille reconduit le portrait négatif que Lucain donnait de César dans $L a$ Pharsale, en particulier dans sa description de sa réaction face à la tête coupée de Pompée. Forestier avance un avis opposé ${ }^{7}$. Selon lui, le César de Corneille n'est pas celui de Lucain : «s'est au contraire imposée au dramaturge la nécessité de laver son héros de toute trace d'ambition factieuse ${ }^{8}$ ». Il considère que Corneille «transforme César en un héros parfaitement vertueux et généreux ${ }^{9} \gg$ et affirme même que Corneille fait donner la description

\footnotetext{
${ }^{1}$ Georges 2006.

${ }^{2}$ Stegmann 1963, 335.

${ }^{3}$ Forestier 2004.

${ }^{4}$ Soit Le Cid, Horace, Cinna et Polyeucte. Voir Ekstein 2003, 270.

${ }^{5}$ Fumaroli 1996, 323-49.

${ }^{6}$ Corneille 1987, 125, cité par Forestier 2004, 249.

${ }^{7}$ Forestier 2004, 248-52.

${ }^{8}$ Ibid., 251.

${ }^{9}$ Ibid., 250.
} 
des réactions de César devant la tête de Pompée par un esclave égyptien pour en discréditer la charge négative ${ }^{10}$.

Notre étude tend à démontrer que La mort de Pompée, loin d'être une pièce composite, est au contraire une construction audacieuse de Corneille qui croise deux intrigues : une égyptienne et une romaine. Ensuite, nous privilégions l'axe d'analyse formé par le couple de César et de Cornélie ; il prolonge la confrontation de César et Pompée et met en évidence l'inachèvement de la guerre civile. Car l'action que nous mettons au cœur de l'étude n'est pas achevée. Plusieurs actions sont accomplies au cours de la pièce. Mais la guerre civile n'est pas achevée et Corneille nous laisse entendre que César ne réussira pas à obtenir la paix qu'il recherche. Enfin, aussi loin de penser que le César de Corneille soit celui de Lucain, comme le propose Fumaroli, que d'admettre qu'il soit un héros parfaitement vertueux, comme l'écrit Forestier, nous montrerons au contraire que le César de Corneille est double, à la fois vertueux et trouble, généreux et conquérant.

\section{Deux tragédies en une}

La mort de Pompée comporte d'une part la tragédie du roi Ptolomée, qui occupe la première partie de la pièce et qui s'accomplit dans l'Acte IV et, d'autre part, la tragédie annoncée de César qui, elle, n'est pas accomplie au sein de la pièce mais rappelée au souvenir du spectateur averti par une série de présages. La première action est moins importante et s'inscrit dans un ensemble plus large qui est celle de la mort annoncée de César. Les deux intrigues se croisent et se cristallisent dans la(les) question(s) : que faire de Pompée (pour Ptolomée) et que faire de la mort de Pompée (pour César).

\subsection{Construction autour d'une absence}

La mort de Pompée s'inscrit dans une série de tragédies françaises écrites dans les années 1630-50 traitant de la mort tragique du personnage principal (Les mort(s) de César, d'Agrippine, des enfans de Brute etc.). Or, la pièce de Corneille présente la particularité de ne pas représenter sur la scène le personnage de Pompée. Son absence de la liste des Acteurs a intrigué plus d'un érudit. La pièce serait-elle un chant funèbre pour Pompée ${ }^{11}$ ou la tragédie annoncée de Jules César? Corneille s'en explique dans son « Examen » de 1660 :

Il y a quelque chose d'extraordinaire dans le titre de ce Poème, qui porte le nom d'un Héros qui n'y parle point ; mais il ne laisse pas d'en être en quelque sorte le principal Acteur, puisque sa mort est la cause unique de tout ce qui s'y passe ${ }^{12}$.

L'absence centrale ${ }^{13}$ de Pompée dans la pièce confirme que le sujet de la tragédie n'est pas la mort de la personne du Grand Pompée mais l'événement politique majeur que constitue son assassinat. Plutôt que la question du héros de la pièce, le titre pointe une crise politique qui oblige les protagonistes à réajuster leur place dans le monde et leurs projets, voire leur(s) politique(s). Certes, la mort de Pompée est «la cause unique » qui précipite Ptolomée vers une mort violente et un événement majeur d'une séquence vers la mort à venir de César. En

\footnotetext{
${ }^{10}$ Ibid., note 67,250 .

${ }^{11}$ Herland 1950, 5.

12 Corneille 1980, 1076.

${ }^{13}$ L'expression est empruntée à Ekstein 2003, 264.
} 
revanche, malgré l'insistance de Corneille sur l'importance du Grand Pompée, « le principal Acteur » de la pièce paraît bien être César.

Corneille a construit sa pièce selon une double problématique, celle des Égyptiens et celle des Romains. L'Acte Ier et l'Acte II sont occupés par Ptolomée et Cléopâtre qui se débattent avec le problème de savoir quel accueil réserver à Pompée qui vient chercher refuge auprès d'eux après sa défaite contre César. Les Actes IV et V sont dominés par les échanges entre les Romains César et Cornélie ${ }^{14}$ et par une mise en avant du problème de la restauration de la paix.

L'Acte III est le point névralgique de la pièce où se déploient les conséquences de l'action des Égyptiens et où se présente à César le problème de savoir que faire de la mort de Pompée. La ligne de partage des eaux se situe dans la question de Charmion, envoyée de Cléopâtre, intéressée par les deux intrigues :

\section{Non, mais elle m'envoie \\ Savoir à cet abord ce qu'on a vu de joie, Ce qu'à ce beau présent César a témoigné, S'il a paru content, ou s'il l'a dédaigné, S'il traite avec douceur, s'il traite avec empire, Ce qu'à nos assassins enfin il a su dire. ${ }^{15}$}

La suite des événements dépendent de la réaction de César. Ptolomée a fait le pari que l'assassinat de Pompée lui serait agréable. Par la narration d'Achorée-principale péripétie et cœur de la pièce-nous saurons que César au contraire prendra le parti d'interpréter cet assassinat comme un attentat contre Rome et réagira en s'emparant de la ville avec ses «trente cohortes » (v. 793). Ptolomée est promptement écarté et son pays colonisé ; le jeune roi mourra en prenant la fuite $(\mathrm{V}, 3)$. Quant à César qui poursuivait Pompée, il doit construire une nouvelle politique face à la disparition de Pompée (qui le réjouit) et face à son assassinat commandité par un roi étranger.

\subsection{Les relations extérieures et les troubles intérieurs}

Corneille aborde la question de la politique extérieure des Romains dans plusieurs de ses tragédies ${ }^{16}$. Il s'en explique dans son avertissement au lecteur de Nicomède :

Mon principal but a été de peindre la politique des Romains au dehors, et comme ils agissaient impérieusement avec les rois leurs alliés, leurs maximes pour les empêcher de s'accroître, et les soins qu'ils prenaient de traverser leur grandeur, quand elle commençait à leur devenir suspecte à force de s'augmenter et de se rendre considérable par de nouvelles conquêtes. ${ }^{17}$

Force est de constater que l'auteur dramatique n'approuve pas la politique conquérante de Rome. La tension entre l'admiration de l'âme romaine et la critique de l'exercice du pouvoir romain vers l'extérieur est au cœur de nombre de ses pièces. Pour saisir les

\footnotetext{
${ }^{14}$ Cléopâtre reste à l'arrière-plan politique dans la deuxième partie de la pièce. Assujettie par César qui avait pris les ports et les portes de la ville égyptienne (Acte III, scène I, v. 794), elle a en outre perdu sa crédibilité pour avoir demandé grâce pour les conseillers conjurés (Acte V, scène V, v. 1426).

${ }^{15}$ Acte III, sc. 1, v. 729-734.

${ }^{16}$ En particulier Polyeucte, La mort de Pompée, Nicomède, Sertorius et Sophonisbe.

${ }^{17}$ Corneille 1984, 641.
} 
préoccupations respectives de Ptolomée et de César, nous avons retenu la notion de politique extérieure avec l'idée que la relation à l'extérieur est un vecteur privilégié de la manifestation du politique. Nous avions à l'esprit la définition du politique, large et à visée comparative, qu'en fait Louis Dumont. Selon lui, c'est dans le rapport d'une société à son extérieur que le politique surgit du social :

[...] le niveau politique apparaît dès qu'une société conçue à l'ordinaire comme multiple se pose comme une face à d'autres (empiriquement comme dans la guerre, ou idéologiquement). La société comme une est ipso facto supérieure à la société comme multiple et la commande légitimement ${ }^{18}$.

Ainsi peut se comprendre l'action de César : une ingérence des alliés dans les affaires internes de Rome lui donne l'occasion de satisfaire le camp de Pompée en assujettissant les étrangers. Il estime que rien n'est plus propice à la cohésion à l'intérieur que la guerre à l'extérieur. Face à l'assassinat de Pompée par les Égyptiens, César se saisit de l'occasion de diriger la haine des Romains contre les Égyptiens dans le but de mettre fin à la guerre civile et rassembler tous les Romains sous lui. Le roi Ptolomée se trouve dans une situation diamétralement opposée par rapport à celle de César. Doublement menacé sur son trône par la conquête romaine et les aspirations de sa sœur qui voudrait partager le pouvoir avec lui selon le vouloir de leur père, il fait le pari de régler les troubles à l'intérieur de son royaume en se faisant de César un allié redevable.

\section{Les perspectives temporelles et les leçons de l'histoire}

La mort de Pompée traite d'un épisode bien connu de l'histoire romaine et qui avait déjà été porté sur la scène par Robert Garnier et Charles Chaulmer ${ }^{19}$. D'un point de vue poétique, la pièce mobilise trois plans historiques : Premièrement, l'action se situe en l'an 48 avant J.C. Deuxièmement, les protagonistes se réfèrent à un moment de leur passé qui est structurant à leurs yeux : d'une part la bataille de Pharsale, rappelée en ouverture de la pièce et, d'autre part, la restauration sur le trône du Ptolomée père par Pompée et César, rappelée par Cléopâtre à son frère le roi qui était trop jeune au moment des faits pour s'en souvenir. Troisièmement, l'avenir des personnages historiques est rappelé à la mémoire des spectateurs : l'assassinat de Jules César en 44 avant J.C. par une conspiration des sénateurs romains qui craignaient que César ne se fasse proclamer roi de Rome, grand tabou depuis la destitution du dernier roi Tarquin. Enfin, mais cela ne nous concernera pas ici, la réception des pièces a lieu dans un quatrième moment, dans le présent du poète dramatique et sa troupe qui s'efforcent de susciter l'intérêt de leurs contemporains. John D. Lyons a identifié ce procédé comme une tragédie des origines. Selon lui, l'historicité des tragédies de Corneille propose une interaction dynamique entre l'histoire et le public :

Within tragedies of origin we find characters who are blinded to the crucial importance of a historic transformation - a new beginning, an origin - by their fixation on an earlier moment that they can recognize as an origin. Thus these tragedies are constructed in such a way that two origins are confronted. The one that lies in the past of the dramatized

\footnotetext{
${ }^{18}$ Dumont 1991, 257.

${ }^{19}$ La Cornélie de Robert Garnier (1574) était bien connue au XVIIème siècle et Corneille semble avoir puisé quelques éléments textuels dans La mort de Pompée de Charles Chaulmer (1638). La tragédie Nouvelle nommée Pompée, publiée en 1579 à Lausanne par un auteur anonyme, n'était probablement pas connue à Paris.
} 
world is not only visible but even sacred to the characters of that world and blinds them to the other, the historic moment they are living. The latter origin is visible to the theatrical audience and provides the basis of the dramatic irony without which no tragedy is possible ${ }^{20}$.

La préscience des spectateurs vis-à-vis des protagonistes procurait une perspective plus large sur les événements qui se déroulaient sur scène. Cela a sûrement contribué à mobiliser la terreur et la pitié des spectateurs-but de la tragédie selon Aristote-de façon particulièrement poignante.

\subsection{Retours en arrière}

La pièce s'ouvre sur le rappel solennel par Ptolomée des atrocités de la bataille décisive de la guerre civile romaine où César a vaincu Pompée. Face à l'arrivée imminente du vaincu qui souhaite se réfugier auprès d'un roi dont il a aidé le père à reconquérir son trône et qui lui est donc redevable, Ptolomée expose le problème qui se présente à lui. Pour lui, c'est sa relation politique avec le vainqueur qui prévaut sur sa dette de reconnaissance à l'égard du vaincu en vertu des conseils de Machiavel aux jeunes princes visant à tenere ou mantenere lo stato $^{21}$ :

\section{Il s'agit de Pompée et nous aurons la gloire D'achever de César ou troubler la victoire, Et je puis dire enfin que jamais potentat N'eut à délibérer d'un si grand coup d'État. ${ }^{22}$}

Ptolomée s'apprête donc à prendre une décision importante. Au dix-septième siècle, un coup d'État désignait « $[\mathrm{u}] \mathrm{n}$ parti vigoureux \& quelquefois violent, qu'une République, une Prince, sont obligés de prendre contre ceux qui troublent l'État» ou « $[\mathrm{u}]$ ne action qui décide de quelque chose d'important pour le bien de 1'État $\gg .{ }^{23}$ Ptolomée aura donc soin de prendre conseil quant à l'attitude à adopter face à une nouvelle configuration du pouvoir romain. Dans une scène de délibération, l'action de ses trois conseillers visera à minorer la dette de reconnaissance du jeune roi vis-à-vis de Pompée. Photin plaide la nécessité absolue de se ranger du côté du vainqueur et de tout faire pour lui plaire, y compris tuer Pompée puisque César le poursuit. Pour convaincre son roi, il énonce un chapelet de maximes d'inspiration opportunistes dont l'emblématique :

Vous ne pouvez enfin qu'au dépens de sa tête [de Pompée]

Mettre à l'abri la vôtre et parer la tempête.

Laissez nommer sa mort un injuste attentat,

La justice n'est pas une vertu d'État. ${ }^{24}$

Le plus modéré Achillas construit un syllogisme pour plaider une action minimale :

- Majeure : «Mais la reconnaissance et l'hospitalité / Sur les âmes des rois n'ont qu'un droit limité »(v. 135-136).

\footnotetext{
${ }^{20}$ Lyons 1996, xiv.

${ }^{21}$ Skinner 1989: 98.

${ }^{22}$ Acte I, sc. I, v. 45-48.

${ }^{23}$ Dictionnaire de l'Académie française (1762), cnrtl.fr/definition/academie4/État

${ }^{24}$ Acte I, sc. I, v. 101-104.
} 
- Mineure : L'aide de Pompée était motivée par un désir de gloire personnelle et n'était de toute façon pas aussi importante que l'assistance de César puisque Pompée avait plaidé pour le rétablissement du roi égyptien alors que César avait financé son retour.

- Conséquence : Pour s'acquitter de sa dette, il suffit de parler pour Pompée, c'est trop de le recevoir. Le grotesque de la chute produit un effet comique en pleine tragédie :

Et si c'est un bienfait qu'il faut rendre aujourd'hui,

Comme il parla pour vous, vous parlerez pour lui.

Quant au conseiller Septime, il feint de peser le pour et le contre d'accueillir Pompée alors qu'en réalité, son argumentation est faussée puisqu'il exclut d'emblée la possibilité de l'accueillir en qualifiant cette option de servitude :

\section{Vous pouvez, comme maître absolu de son sort, \\ Le servir, le chasser, le livrer vif ou mort. \\ Des quatre le premier vous serait trop funeste ; \\ Souffrez donc qu'en deux mots j'examine le reste. ${ }^{25}$}

Les sophismes des conseillers achèveront de convaincre Ptolomée qui prendra la décision de faire assassiner Pompée :

N'examinons donc plus la justice des causes,

Et cédons au torrent qui roule toutes choses. ${ }^{26}$

Le torrent peut désigner la confluence des arguments des conseillers ou la fortune de César. Cela paraît surtout désigner le libre cours de la passion de la vengeance, la rancœur des États alliés soumis :

Secondons le destin qui les veut mettre aux fers [les Romains], Et prêtons-lui la main pour venger l'univers. ${ }^{27}$

Ainsi, le jeune roi croit voir sa rancune dans la volonté du destin et choisira d'ignorer les avertissements de sa sœur qui lui explique qu'il doit son trône à l'action de Pompée auprès de César. La tirade de Cléopâtre dans la deuxième partie de l'Acte Premier rétablit-face à Ptolomée et aux spectateurs-la dette de reconnaissance que les conseillers avaient minorée. Le choix de Ptolomée qui cède à son désir de vengeance pour les humiliations subies de la part des Romains trouvera écho plus tard dans la pièce chez César qui n'entendra pas les avertissements de Cornélie. Dans la mesure où ces choix les conduiront tous deux respectivement à leur perte, Corneille semble suggérer que le non-respect ou la méconnaissance de l'histoire est une faute en politique.

\subsection{Présages}

La mort de Pompée est la tragédie annoncée de Jules César, mettant en évidence l'échec de ses projets d'avenir. Son assassinat futur est rappelé au souvenir du spectateur par le biais de deux présages :

\footnotetext{
${ }^{25}$ Acte I, sc. I, v. 163-166.

${ }^{26}$ Acte I, sc. I, v. 189-190.

${ }^{27}$ Acte I, sc. I, v. 199-200.
} 


\begin{abstract}
Ainsi finit Pompée et peut-être qu'un jour
César éprouvera même sort à son tour.

Rendez l'augure faux, Dieux qui voyez mes larmes ${ }^{28}$
\end{abstract}

Le changement de rythme, provoqué par l'effroi de Cléopâtre lorsqu'elle s'entend prononcer le présage suite à sa réflexion méditative sur l'instabilité du monde appelle l'attention du spectateur ainsi que l'usage du mot « augure ». Quant au présage de Cornélie à la fin de la pièce, il se fait plus précis :

\title{
Mais sache aussi qu'alors la jeunesse romaine \\ Se croira tout permis sur l'époux d'une reine, \\ Et que de cet hymen tes amis indignés \\ Vengeront sur ton sang leurs avis dédaignés. ${ }^{29}$
}

En effet, les sénateurs romains s'uniront pour poignarder César parce qu'il s'était fait nommer dictateur à vie et parce qu'ils craignaient l'instauration d'une monarchie héréditaire. Les présages de Cléopâtre et de Cornélie jettent une lumière ironique sur les projets des protagonistes qui ignorent leurs échecs futurs. La mise en perspective temporelle des tableaux historiques fait surgir sur la scène une relation de causalité que les protagonistes doivent impérativement prendre en compte si leur politique doit réussir. Ce rappel au futur passé ${ }^{30} \mathrm{de}$ la chute de César alors que l'action se déroule à la veille de la fondation du pouvoir impérial sur les ruines de la République, pouvait avoir des résonnances toutes particulières pour les intellectuels du dix-septième siècle ayant en mémoire les guerres de religions qui avaient divisé le pays une génération plus tôt. En effet, la conception du temps et de l'histoire qui prévalait encore à l'époque moderne selon Reinhardt Koselleck (2004) voyait une exemplarité dans les événements historiques se ressemblant. Dans une conception du monde où le cours du temps est limité à l'horizon par l'apocalypse, une guerre civile ressemble à une autre, même si les contextes sont différents. Ainsi, le rappel de l'assassinat de César pouvait profondément toucher un public habitué à considérer l'histoire comme porteuse de leçons de vie pour l'avenir.

\section{Réaction(s) de César face à la tête de Pompée}

Situé en ouverture de l'Acte III, la narration d'Achorée qui raconte à la suivante de Cléopâtre la réaction de César à l'offrande de Ptolomée constitue le nœud de la pièce, juste avant l'entrée en scène de César et de la mise en œuvre de sa politique vis-à-vis de l'Égypte. Dans son «Examen », Corneille précise qu'il a puisé ses sources pour cette scène chez Plutarque et Lucain. Il est utile de comparer ce que l'un et l'autre écrivent pour saisir les choix qui ont régi la composition de Corneille.

\subsection{Choix de Corneille par rapport aux sources}

La description de Plutarque est la plus courte et la plus simple ; il est question de deux étapes de la découverte, entraînant deux réactions opposées :

\footnotetext{
${ }^{28}$ Acte II, sc. II, v. 587-589.

${ }^{29}$ Acte V, sc. IV, v. 1749-1752.

30 Terme emprunté à Koselleck 2004.
} 
Telle fut la fin de Pompée. Peu après, César arriva dans cette Égypte qui avait été infectée d'une si grande souillure. Quelqu'un lui présenta la tête de Pompée, mais il se détourna de cet homme comme d'un maudit. Cependant, il accepta le sceau de Pompée et se mit à pleurer en y voyant gravé un lion armé d'une épée ${ }^{31}$.

La découverte de la tête suscite la répulsion; les signes de Pompée entraînent les pleurs. Ce passage est neutre à l'égard de César, strictement restreint à une description de deux séquences comportementales. En revanche, Lucain propose une description plus riche, psychologique et ironique, en affichant sa sympathie républicaine :

Ce n'est pas au premier aspect que César réprouve le présent (la tête de Pompée) et qu'il en détourne les yeux : il y attache ses regards, jusqu'à ce qu'il n'ait plus un doute ; dès qu'il a vérifié le crime et qu'il croit désormais pouvoir sans danger montrer la générosité d'un beau-père, il répand des larmes contraintes, et fait sortir des plaintes de son cœur satisfait, incapable de déguiser autrement que par des larmes les sentiments de joie qu'il a laissé paraître ${ }^{32}$.

Le César de Lucain est un hypocrite, un calculateur qui déguise ses sentiments. Les deux étapes sont dans un ordre inverse de celui de Plutarque : César s'assure d'abord qu'il s'agit bien de la tête de son ennemi mort, puis feint la générosité en cachant par les larmes sa joie d'avoir vaincu. Dans sa tragédie, Corneille fait un choix différent de ceux de Plutarque et de Lucain. Sa description est moins plate que celle de Plutarque et introduit, comme Lucain, une analyse psychologique des réactions successives de César. Mais contrairement à Lucain, dont il dit admirer les vers, la complexité des mouvements du vainqueur n'est pas celle de l'hypocrisie. En effet, face à la tête de son principal ennemi, César est tout d'abord traversé par un mouvement premier de jubilation victorieuse. La vue de la preuve concrète de sa victoire, fournie indépendamment de sa volonté, déclenche en lui un processus où son penchant naturel est bientôt combattu par son esprit et par sa vertu, processus qui le conduira à adopter l'indignation, la défiance à l'égard de Ptolomée, et la prise du port et de la ville. Enfin, Corneille introduit une séquence extraordinaire où se succèdent le regard, l'examen intérieur, les larmes, les gestes, les ordres et les actions par lesquels, face au choc, la détermination tourmentée de la vertu se transforme en politique.

Le soin que Corneille a consacré à cette scène ne témoigne pas seulement de son importance pour l'histoire et pour la tragédie. La complexité qu'il y a introduite indique l'enjeu pour la composition de la pièce de la définition du caractère de César. Il doit être double, mais cette duplicité doit être une tension interne plutôt qu'une simple hypocrisie.

\subsection{La narration d'Achorée}

Corneille dit dans son «Examen » de la pièce que la narration d'Achorée du troisième acte est «à [s]on gré la plus magnifique ${ }^{33} »$. La règle de la bienséance interdisant d'apporter du sang sur le théâtre, la scène doit être rapportée avec un décalage dans le temps, par des personnes «qui ont l'esprit assez tranquille pour avoir toute la patience qu'il y faut donner $^{34} »$, qui ont donc une distance par rapport aux événements. Cette patience permet de

\footnotetext{
${ }^{31}$ Plutarque 2001, 1218-1219.

${ }^{32}$ Corneille 1980, « Notes et variantes », 1742.

${ }^{33}$ Corneille 1963, 317.

${ }^{34}$ Corneille 1963, 316-3017.
} 
décortiquer le processus qui va du choc initial à l'action en passant par la délibération, analyse d'une prise de décision politique. En effet, passé l'effet de surprise, écartée la douceur de la victoire sur son adversaire, César prend la décision de laisser couler ses larmes montrant par là une prise de position politique, basé sur la «vertu »: Il faut pleurer Pompée, parce qu'il était un grand consul romain, assassiné en terre étrangère, par des étrangers qui lui étaient redevables. Il se positionne en homme d'État qui pleure un autre grand homme d'État. Alors que les larmes ne sont pas considérées viriles, elles sont ici requises par «la signification publique de la mort ${ }^{35} »$. Lorsqu'un grand homme d'État romain est assassiné par des étrangers, c'est Rome qui subit un affront. En l'espace de quelques instants, César engage une manœuvre visant à rétablir l'unité des Romains. Il marque son indignation à l'égard des Égyptiens, force ses hommes à une minute de silence avant de lancer un assaut militaire sur la ville du roi égyptien. Sa prise de position est rendue publique par un comportement ostentatoire propre à diffuser le message d'un changement de situation, d'une nouvelle politique vis-à-vis de l'Égypte. Ainsi, César

Fait valoir sa défiance, ainsi que ses regrets,

Parle d'Égypte en maître et de son adversaire,

Non plus comme ennemi, mais comme son beau-père ${ }^{36}$.

Comme l'indiquent également les sources historiques, César souhaitera apaiser les Romains divisés, pardonner à ceux qui ont suivi son adversaire et rétablir la paix civile une fois remportée la victoire :

Et mon ambition ne va qu'à les forcer,

Ayant dompté leur haine, à vivre et m'embrasser ${ }^{37}$.

Ainsi, le nœud de la pièce est le moment où le vainqueur de la guerre civile reste seul face au cadavre du vaincu. Que va-t-il faire de la mort de Pompée ? César choisira d'adopter une politique de rassemblement des Romains dans une démarche de rétablissement de la paix civile. Or, la veuve de Pompée va lui opposer son devoir de vengeance.

\section{Cornélie et César}

Dans son «Examen » de la pièce, Corneille précise qu'il n'a ajouté à l'histoire que ce qui concerne Cornélie ; qu'il a choisi de « feindre [...] qu'elle fut ramenée devant César, bien que l'histoire n'en parle pas $^{38} »$. Contrairement à un Sévère dans Polyeucte ou à une Eryxe dans Sophonisbe, personnages inventés par Corneille et qui expriment un point de vue politique ou une attitude qui contribue de façon déterminante au dénouement de la pièce, Cornélie est un personnage historique ; c'est sa présence et sa confrontation avec César qui est l'invention de Corneille. Or, il convient de porter une attention toute particulière à tout écart entre l'histoire réelle et l'intrigue forgée par Corneille. S'il ménage une confrontation entre César et Cornélie qui n'a pas eu lieu, c'est qu'elle sert son dessein de dramaturge. Par conséquent, il faut considérer la présence des Cornélie comme un révélateur des enjeux de la pièce.

\footnotetext{
${ }^{35}$ Merlin-Kajman 2000, 130-31.

${ }^{36}$ Acte III, scène I, v. 796-98.

${ }^{37}$ Acte III, scène II, v. 919-20.

${ }^{38}$ Corneille 1963, 316.
} 


\subsection{Cornélie : un obstacle à la politique de César}

Toutes les entrées sur scène de Cornélie constituent des obstacles aux projets de César-projets amoureux mais surtout politiques. Sa première entrée en scène survient au moment où César s'apprêtait à rejoindre Cléopâtre. Le comique de sa réaction contraste avec le ton du poème, surtout dans celui dont Corneille avait dit lui-même que le style était «plus élevé [...] qu'en aucun des miens et ce sont, sans contredit, les vers les plus pompeux que j'aye faits ${ }^{39} \gg$. Les accents pastoraux n'en sont que plus savoureux et ne laissent pas de rappeler-par touches plus délicates, certes-la pièce homonyme de Chaulmer ${ }^{40}$ :

Qu'elle entre. Ah! l'importune et fâcheuse nouvelle!

Qu'à mon impatience elle semble cruelle !

$\mathrm{O}$ ciel ! et ne pourrai-je enfin à mon amour

Donner en liberté ce qui reste du jour $?^{41}$

Alors que Cornélie l'avertit qu'elle n' «abaisse[ra] pas [s]a haine », César ne voit en elle que la grandeur romaine et lui promet la paix et la liberté. Elle fait une deuxième entrée en scène fulgurante, interrompant son entretien amoureux avec Cléopâtre, pour avertir César d'une conspiration d'assassinat contre lui et lui réitérer ses menaces.

L'Acte $\mathrm{V}$, la scène finale exceptée, est dominé par la présence de Cornélie. La première scène l'établit comme l'égale de César, elle dit comprendre le raisonnement et les motivations de César («au point qu'il en est, j'en voudrais faire autant ${ }^{42} »$ ). Ensuite, sa présence sert à tempérer l'optimisme ambiant jusqu'à sa tirade finale où elle promet de soulever contre César le parti pompéen et termine, en point d'orgue, par le présage de son assassinat à Rome.

\subsection{De l'impossibilité de rétablir la paix civile}

Dans une note de bas de page de son édition des œuvres de Corneille, André Stegmann rappelle que La mort de Pompée appartient au genre que les Italiens ont nommé «tragédie à fin heureuse ${ }^{43} \gg$. Cela est vrai de l'une des deux fins de la tragédie, celle de l'épisode relatée par la pièce: la présentation de la reine Cléopâtre à son peuple, au bras de César. Mais l'allégresse de la scène finale sonne creux et le spectateur sait que ce moment heureux n'est qu'un répit et que la suite de l'histoire ne sera pas heureuse. Ainsi, l'ajout du personnage de Cornélie à l'intrigue et son projet de reconduire la guerre civile à l'encontre de César pour venger son époux met en évidence la futilité du désir de César de «vaincre et pardonner ${ }^{44}$ ». Ainsi est posée la question de la difficulté du rétablissement de la paix civile.

\section{Conclusion}

A l'horizon temporel de la pièce-rappelé par les présages de Cornélie et de CléopâtreCésar sera assassiné en sa qualité de futur tyran, trop puissant pour diriger Rome. Ici,

\footnotetext{
${ }^{39}$ Corneille 1963, 316.

${ }^{40}$ La tragédie La mort de Pompée de Charles Chaulmer, publiée en 1938, est composée comme un mélange de genres dramatiques.

${ }^{41}$ Acte IV, scène III, v. 977-80.

${ }^{42}$ Acte V, scène I, v. 1556.

${ }^{43}$ Corneille 1963, note $21,335$.

${ }^{44}$ Acte III, scène II, v. 916.
} 
Corneille met en avant la menace que constitue la conquête impériale à l'intérieur. Pour bien le comprendre, il faut comparer César avec un autre grand conquérant romain, Scipion l'Africain. César aurait souhaité faire une entrée triomphale à Rome sur le même char que Pompée vaincu ${ }^{45}$. Il pense qu'il peut tout faire par sa seule volonté, éteindre la haine, soulager les rancœurs, faire oublier les deuils. Il croit qu'il peut apporter la paix : c'est le contraire qui se produit. Le destin de César peut être mis en contraste avec Scipion, tel qu'il est évoqué par le stoïcien Sénèque ${ }^{46}$. Scipion, soupçonné de vouloir se faire roi ou tyran à Rome, s'est retiré dans sa villa près de Naples et meurt en exil :

[...] c'est sa noble modération, sa piété patriotique, encore plus admirable, à mon sens, quand il renonça à sa patrie que lorsqu'il la défendit. Ou Scipion devait être perdu pour Rome ou Rome perdre ses libertés. «Loin de moi, dit-il, la pensée de toucher aux lois, aux institutions. Que l'égalité demeure entière entre tous les citoyens. Jouis sans moi, ô ma patrie, du bienfait que tu as reçu de moi. J'ai été l'agent effectif de ton indépendance, je serai aussi la preuve effective de ta liberté : je pars, si tu estimes que j'ai atteint plus haut qu'il ne te convient. » Comment n'admirerais-je pas une pareille grandeur d'âme ? Il s'exile volontairement, il soulage la cité de sa présence. Les choses en étaient venues à ce point, que la liberté devait attenter contre Scipion ou Scipion attenter à la liberté. Attentat sacrilège des deux parts! Il s'effaça donc devant les lois et choisit pour retraite Literne, voulant que l'État lui fût autant redevable de son propre exil que de l'exil d'Hannibal. ${ }^{47}$

Les deux consuls conquérants sont devenus trop grands pour Rome, une menace pour le pouvoir en place. L'aîné a eu la prudence de se retirer, son successeur non. La conquête fait trembler le pouvoir établi ; la fortune des deux conquérants a atteint un point de limite d'où il faut se retirer ou mourir.

Le choix de Scipion l'Africain, relaté par Sénèque, et le destin tragique de César disent l'impossibilité de concilier la conquête et le règne. Sénèque admirait Scipion pour l'avoir compris, Corneille dit la même impossibilité en montrant la naïveté de César de vouloir « vaincre et pardonner».

\section{Bibliographie}

Corneille, Pierre. 1963. Euvres complètes, édition d'André Stegmann. Paris : Éditions du Seuil, collection Intégrales.

Corneille, Pierre. 1980 (tome I), 1984 (tome II), 1987 (tome III). Cuvres complètes, Textes établis, présentés et annotés par Georges Couton. Paris : Gallimard, Bibliothèque de la Pléiade.

Dumont, Louis. 1991. Homo aequalis, II. L'idéologie allemande. France-Allemagne et retour. Paris : Gallimard.

Ekstein, Nina. 2003. "Pompée's Absence in Corneille's La mort de Pompée ». Rivista di letturature moderne e comparate, 56, 259-273.

Forestier, Georges. 2004. Essai de génétique théâtrale. Corneille à l'œuvre. Genève : Droz.

\footnotetext{
${ }^{45}$ Acte III, scène II, v. 923-24.

${ }^{46}$ Corneille était un lecteur de Sénèque selon Fumaroli 1996, 8 et passim.

${ }^{47}$ Sénèque 1993, 867-68.
} 
Fumaroli, Marc. 1996. Héros et orateurs. Rhétorique et dramaturgie cornéliennes. Genève : Droz.

Georges, André. 2006. «La mort de Pompée est-elle une tragédie ? Lettres romanes, vol. 60, issue 3-4, 173-80.

Herland, Louis. 1950. "Les éléments précornéliens dans La mort de Pompée de Corneille. » Revue d'Histoire littéraire de la France, 50e Année, No. 1. 1-15

Koselleck, Reinhardt. 2004. Futures Past. On the Semantics of Historical Time. New York: Columbia University Press.

Lyons, John D. 1996. The Tragedy of Origins. Pierre Corneille and Historical Perspective. Stanford : Stanford University Press.

Merlin, Hélène. 1994. Le public et la littérature au XVIIe siècle, Paris : Les Belles Lettres.

Merlin-Kajman, Hélène. 2000. L'absolutisme dans les lettres et la théorie des deux corps. Passions et politique. Paris : Honoré Champion.

Plutarque. 2001. Vies parallèles, édition de François Hartog. Paris : Gallimard, collection Quarto.

Prigent, Michel. 2008. Le héros et l'État dans la tragédie de Pierre Corneille. Paris : PUF/Quadrige.

Sénèque. 1993. Entretiens. Lettres à Lucilius, édition de Paul Veyne. Paris : Roger Laffont/Bouquins.

Skinner, Quentin. 1989. « The State. » In Terence Hall, James Farr et Russell L. Hanson (éd.). Political Innovation and Conceptual Change. Cambridge: Cambridge University Press, 99-131.

Thuau, Étienne. 2000. Raison d'État et pensée politique à l'époque de Richelieu. Paris : Albin Michel. 